\title{
Numerical Simulation on Emulsion Spray Flow Field at Different Installation Torsion Angles
}

\author{
Haijun Qiao ${ }^{1, a}$, Jianan $\mathrm{Wu}^{2, \mathrm{~b}}$ \\ ${ }^{1}$ Yanshan University, Qinhuangdao, 066004, China; \\ ${ }^{2}$ Dept. of Common Courses, Hebei University of Environmental Engineering, Qinhuangdao, 066102, China. \\ aqiaohaijun@ysu.edu.cn, bbrainbow@sohu.com
}

Keywords: Numerical simulation, emulsion, spray, flow field, installation torsion angle, distribution.

\begin{abstract}
The emulsion spray system in the strip steel cold rolled machine cleans the working roll surfaces during rolling to improve the quality of the rolled steel plate. Emulsion spray flow fields was physically modelled and numerically simulated. The distribution of multi-nozzle emulsion flow field, its overlapping region and optimum installation torsion angle were obtained, which could lay a foundation for making further improvement on performance of the emulsion spray system.
\end{abstract}

\section{Introduction}

The emulsion spray flow field of the cold rolled strip tempering machine can clean the working roll surface during rolling, therefore improving the rolled plate shape and reducing the roll surface wear.

The emulsion spray flow field of the cold rolled strip tempering machine can clean the working roll surface during rolling, therefore improving the rolled plate shape and reducing the roll surface wear.

The numerical simulation on the flow field of the emulsion spray was mainly focused on the high pressure jet.

G.J. Li et al. [1, 2] studied the high-speed impinging stream of ultra-high pressure emulsion at 300 MPa pressure. The SIMPLE algorithm and the velocity field and turbulent kinetic energy distribution in the jet zone were used.

X. B. Zhu et al. [3] simulated and experimentally studied on external velocity and pressure field of high-pressure nozzle jet for phosphorus. They use unstructured hybrid grid with VOF (Volume of Fraction) unsteady flow model and PISO algorithm under the condition of $20 \mathrm{MPa}$ on the nozzle entrance; jet velocity near target distance in the phosphorus removal nozzle jet flow field was analyzed.

G. L. Yang et al. [4-6] applied the CFD technique to numerically simulate the incompressible single phase jet inside and outside a convergent, conical, or rotating nozzle. Most of their researches use SIMPLY or SIMPLIC algorithm and K-Epsilon or RNG K-Epsilon two equation turbulence model to explore the simplified two-dimensional physically modelled and unstructured meshed velocity, pressure field and turbulent kinetic energy distribution inside and outside of the nozzles.

The research simulates the complex three-dimensional gas-liquid two-phase flow field of the emulsion jet, from flat nozzle entrance to the surface of the steel plate, analyzes the three-dimensional flow characteristics inside and outside the emulsion nozzle, the velocity and pressure under different installation torsion angle, provides theoretical and numerical basis for the design, selection and optimization of wet emulsion system. 


\section{Numerical Simulation on Flow Field of Emulsion Spray of Multi-Nozzles with Different Rotating Positions}

Physical Modeling. The physical and mathematical model of the multi-flat nozzles flow field is established with Pro/Engineering, including two nozzles, the roll surface, the surrounding environment and the artificial interface, which calculating area size is $306 \mathrm{~mm} \times 200 \mathrm{~mm} \times 340 \mathrm{~mm}$.

Because the flow field distribution of each nozzle is approximately the same, the flow field distribution in the overlap area between nozzles is almost the same. So in the analysis of the emulsion flow field, only two adjacent nozzles are chosen, physically modeled and numerically simulated. The emulsion flow field of the multi-nozzles is shown in Fig. 1 below.

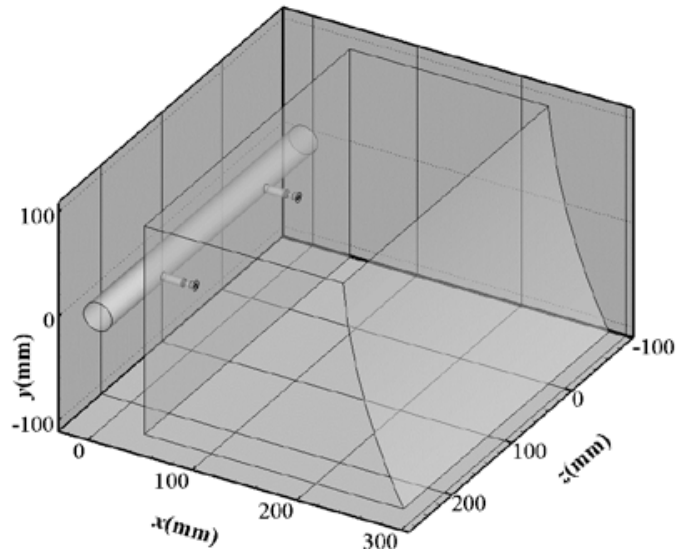

Fig. 1 Model of the multi-nozzles emulsion flow field

Solver Settings. Steady state and implicit pressure based solver and K-epsilon two equation model were used. The coupling of pressure and velocity took SIMPLE algorithm. VOF two phases flow model was taken to track the free surface of jet, with air as the main phase, and water the auxiliary phase.

The type of boundary conditions for one end of the spray beam entrance is pressure inlet, 5bar. The inner surface of the beam and the nozzles corresponding with adopt the fixed-wall type. The injection areas around the top and bottom of the nozzles adopt pressure outlet boundary conditions.

It is assumed that the emulsion was incompressible, i.e., the density does not vary with the motion and remains constant.

Mesh generation. The Fig. 2 below dedicates the gridding at each installation torsion angle $(\alpha)$ of multi-nozzle flow field.

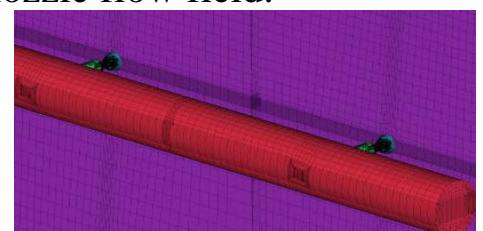

(a) $\alpha=5^{\circ}$

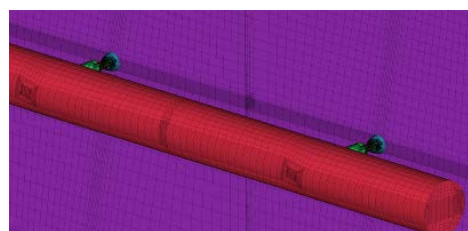

(b) $\alpha=10^{\circ}$

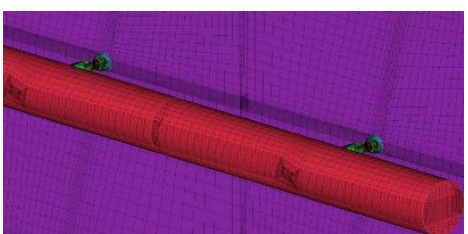

(c) $\alpha=15^{\circ}$

Fig. 2 Grids of multi-nozzles emulsion flow field at different installation torsion angles The number of meshes is up to 460000 , hexahedral.

\section{Results and Discussion}

Fig. 3 and Fig. 4 show the velocity contour and the vector diagram of some typical positions of the installation torsion angle between 0 and 25 degrees, respectively. 


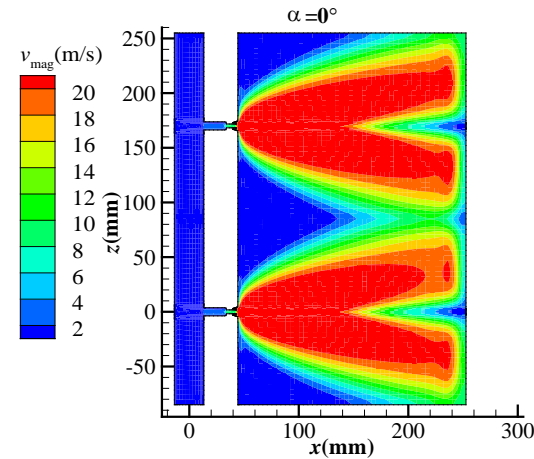

(a) $\alpha=0^{\circ}$

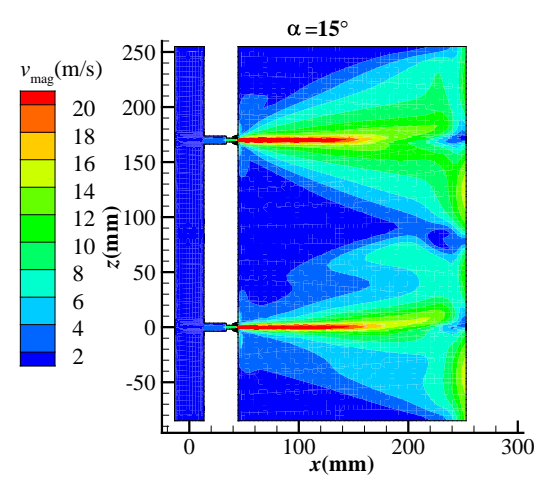

(d) $\alpha=15^{\circ}$

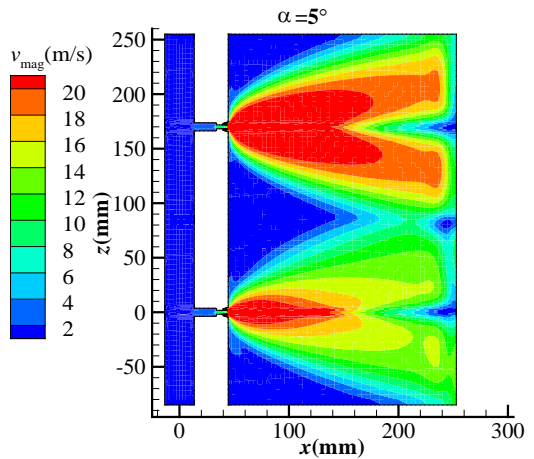

(b) $\alpha=5^{\circ}$

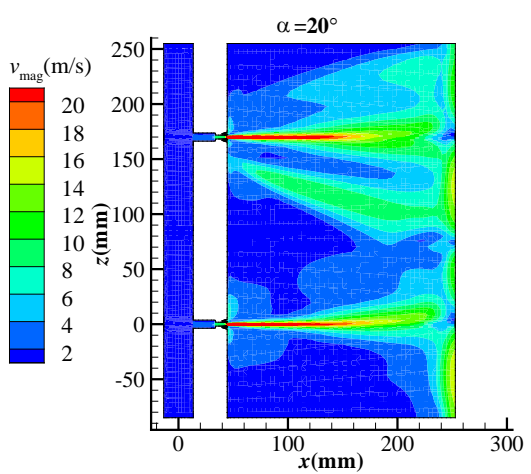

(e) $\alpha=20^{\circ}$

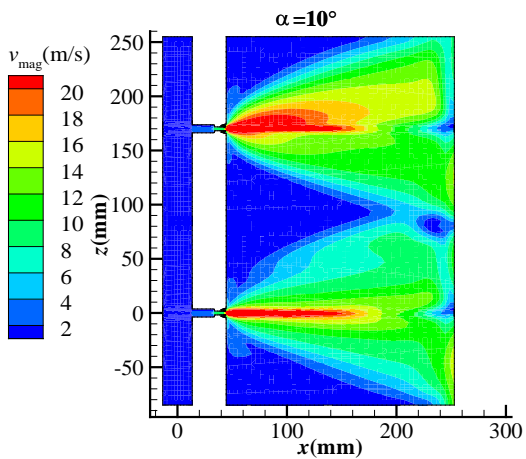

(c) $\alpha=10^{\circ}$

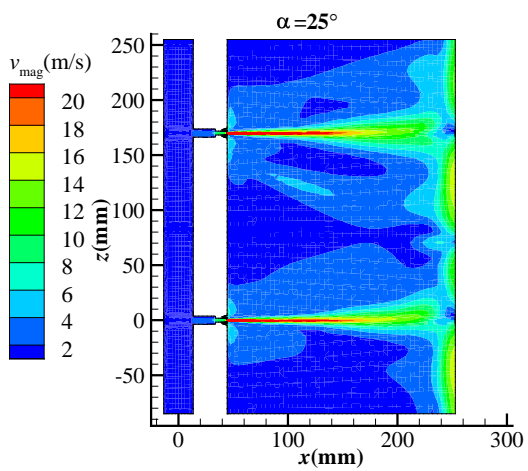

(f) $\alpha=25^{\circ}$

Fig. 3 Velocity contour of multi-nozzles emulsion flow field at different installation torsion angles

As can be seen from the diagrams, although the two nozzles are identical and the meshing conditions are eventually basically the same, the velocity contours are not exactly the same. It's due to the flow resistance losses caused by the internal passage of the nozzle beam.

It can be seen that with the increase of installation torsion angles, two distinct vortices are gradually formed between the two nozzles. There is almost no overlap interference at the 2 nozzle exit under small installation torsion angles, which can also be seen from the vector diagram of the multi-nozzle jet flow field in the Fig. 4.

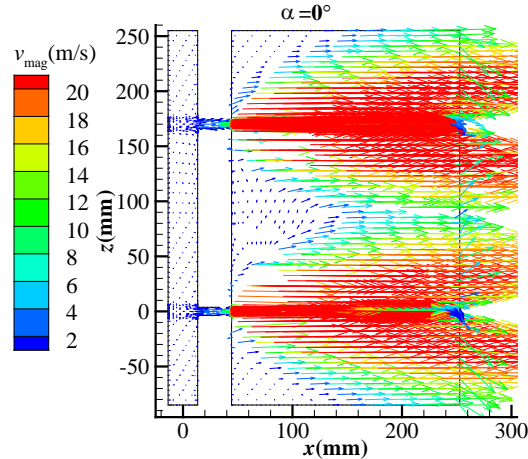

(a) $\alpha=0^{\circ}$

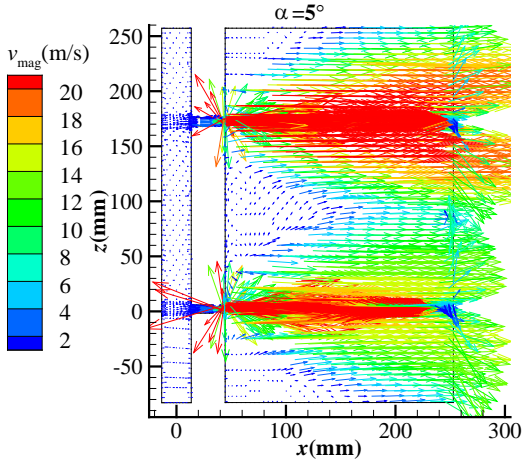

(b) $\alpha=5^{\circ}$

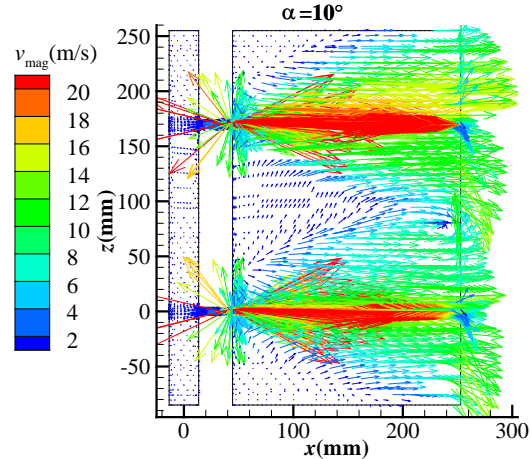

(c) $\alpha=10^{\circ}$ 


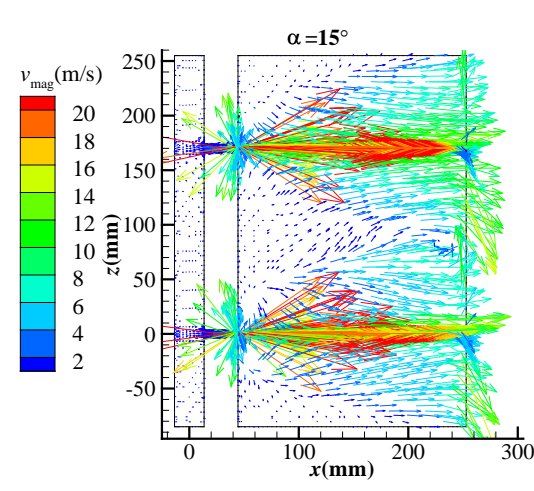

(d) $\alpha=15^{\circ}$

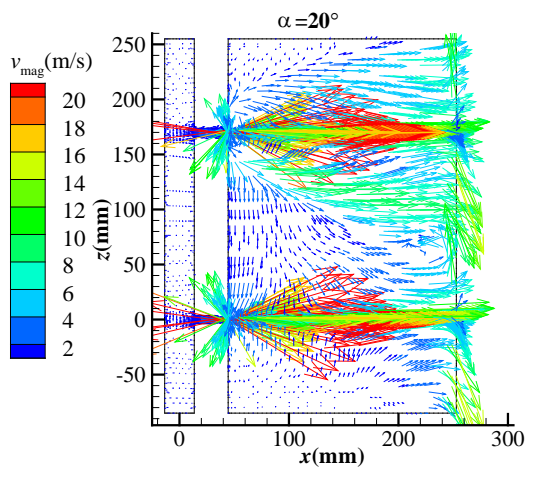

(e) $\alpha=20^{\circ}$

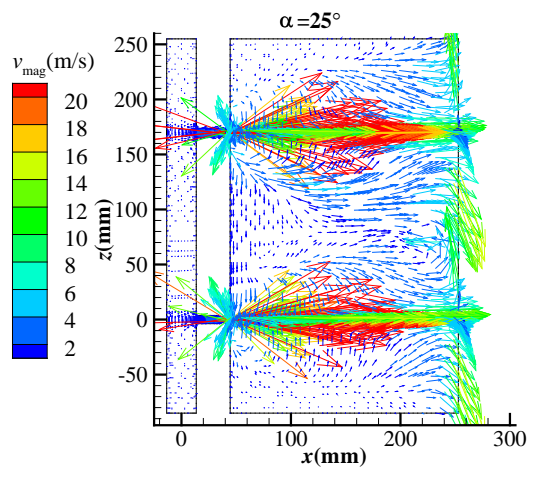

(f) $\alpha=25^{\circ}$

Fig. 4 Velocity vector of multi-nozzles emulsion flow field at different installation torsion angles

With the increase of installation torsion angles, the vortex range and flow velocity between 2 nozzles increase, which is beneficial to the uniformity of emulsion distribution on the roll surface. On this point of view, it is advantageous to increase the installation torsion angles. But the large installation torsion angles will reduce the kinetic energy impacted on the $y=0$ cross section, so the optimum installation torsion angles is moderate, that is 15 and 20 degrees.

\section{Summary}

Based on physical modeling, mesh generation and numerical calculation of emulsion jet flow field and corresponding roll surface, the external three-dimensional multi-nozzle emulsion flow fields at 0-25 degree installation torsion angles on a cold rolled steel plate tempering machine were modelled and numerical simulated. The two-phase velocity field and the distribution law of overlap region of multi-nozzle emulsion flow field were obtained.

Considered the uniformity of emulsion distribution and the kinetic energy of spray, the 15-20 degrees are the optimum torsion angle. The results provide a powerful reference for ensuring the reasonable distribution of liquid and ensuring the rolling quality of strip steel.

\section{References}

[1] G.J. Li, J.Z. Pan, Academic analysis of numerical simulation of emulsification nozzle by super high pressure. J. of East China University of Science and Technology (Natural Science Edition). 34 (2008) 144-148.

[2] G.J. Li, J.Z. Pan, Optimized analysis for impinging streams emulsification nozzle under hyper high pressure on software fluent, Chinese Quarterly of Mechanics. 29 (2008) 634-641.

[3] X.B. Zhu, K.S. Chen, Y. Wang, The simulation and experimental study on jet field of high-pressure nozzle for phosphorus, Machinery Design \& Manufacture, 5 (2009) 217-219.

[4] G.L. Yang, X.H. Li, W.H. Zhou. The influence of conical nozzle geometric parameters on the property of efflux flow field, Chinese Hydraulics \& Pneumatics, 5 (2009) 62-64.

[5] G.L. Yang, L. Chen, X.H. Li, The influence of conical nozzle geometric parameters on the property of efflux flow field. Chinese Hydraulics \& Pneumatics, 11 (2009) 6-8.

[6] G.L. Yang, W.H. Zhou, F. Liu, Simulation of flow field of high-pressure water-jet from nozzle with FLUENT, J. of Lanzhou University of Technology, 34 (2008) 49-52. 